\title{
Evolutionary Generation of Prototypes for a Learning Vector Quantization Classifier
}

\author{
L. P. Cordella ${ }^{1}$, C. De Stefano ${ }^{2}$, F. Fontanella ${ }^{1}$ and A. Marcelli ${ }^{3}$ \\ 1 Dipartimento di Informatica e Sistemistica \\ Università di Napoli Federico II, \\ Via Claudio, 2180125 Napoli - Italy \\ \{cordel, frfontan\}@unina.it \\ 2 Dipartimento di Automazione, Elettromagnetismo, Ingegneria dell'Informazione e \\ Matematica Industriale \\ Università di Cassino \\ Via G. Di Biasio, 4302043 Cassino (FR) - Italy \\ destefano@unicas.it \\ 3 Dipartimento di Ingegneria dell'Informazione e Ingegneria Elettrica \\ Università di Salerno \\ 84084 Fisciano (SA) - Italy \\ amarcelli@unisa.it
}

\begin{abstract}
An evolutionary computation based algorithm for data classification is presented. The proposed algorithm refers to the learning vector quantization paradigm and is able to evolve sets of points in the feature space in order to find the class prototypes. The more remarkable feature of the devised approach is its ability to discover the right number of prototypes needed to perform the classification task without requiring any a priori knowledge on the properties of the data analyzed. The effectiveness of the approach has been tested on satellite images and the obtained results have been compared with those obtained by using other classifiers.
\end{abstract}

\section{Introduction}

Classification problems are probably among the most studied ones in the field of computer science since its beginnings [1]. Classification is a process according to which an object is attributed to one of a finite set of classes or, in other words, it is recognized as belonging to a set of equal or similar entities, identified by a name (label in the classification field jargon). In the last decades, Evolutionary Algorithms (EAs) have demonstrated their ability to solve hard non linear problems characterized by very complex search spaces [2]; they have also been used to solve classification problems.

Genetic Algorithms (GAs), for example, have been widely applied for evolving sets of rules that predict the class of a given object. According the GAs-based approach, referred to as learning classifier systems (LCS) [3], the individuals in the population encode one or more prediction rules of the form IF-THEN, 
where the antecedent part of the rule contains a combination of conditions on some attributes of the patterns to be classified, while the consequent part rule expresses the class predicted by the rule. GAs for rule discovery can be divided into two main classes, depending on how rules are encoded by individuals. The classes are referred to as Michigan $[4,5]$ and Pittsburgh $[6,7]$. The former approach employs a single individual for encoding one prediction rule, whereas in the latter approach each individual encodes a whole set of rules. Both these approaches were originally devised to solve single-class problems. Successively, also multi-class problems have been tackled. These kind of problems have been faced by introducing multiple populations, so that each population evolves the rule of a specific class. However, in this way it is hard to model the interaction between the rules representing different classes when new and unknown patterns have to be recognized. Moreover, another problem that affects the LCS algorithms is due to the difficulty of solving the conflict that comes up when single pattern is matched by several rules, each predicting a different class. As regards the GP-based methodologies, only recently they have been proposed to solve classification problems [8-10]. In [9], for example, a GP system has been devised to evolve equations involving simple arithmetic operators and feature variables, for hyper-spectral image classification. In [8], a GP approach has been employed for image classification problems, adding exponential functions, conditional functions and constants to the simple arithmetic operators. GP has also been used to evolve sets of fuzzy rules [11]. In [10], an interesting method, which considers a $n$-class problem as a set of two-class problems, has been introduced. When the expression for a particular class is searched, that class is considered as target, while the other ones are merged and treated as a single undesired class. In all approaches mentioned above, the number of prototypes to be used to solve the classification problem at hand is set exactly equal to the number $n$ of classes to be dealt. Consequently, these approaches do not consider the existence of subclasses within one or more of the classes in the analyzed data set: in practice, each of these subclasses needs a specific prototype to be represented.

We propose a new classification method, based on the concept of Learning Vector Quantization (LVQ). Given a data set for which the patterns are feature vectors, this algorithm provides a set of reference vectors, to be used as prototypes for the classification task to be performed. The effect of any LVQbased algorithm is a Voronoi tessellation of the feature space in which the data are represented. In practice, LVQ allows one to partition the feature space into a number of regions, each identified by one of the vectors provided after the training phase. Such regions are bordered by hyperplanes defined as the loci of points that are equidistant from the two nearest reference vectors. The reference vectors represent the prototypes of the points inside the regions and each region represents a cluster. The classification method proposed here uses a specifically devised evolutionary algorithm for evolving variable size sets of feature vectors. The remarkable feature of our approach is that it does not require any a priori knowledge about the actual number of prototypes, i.e. reference vectors, each class needs to be represented. 
The proposed classification method has been tested on satellite images and the results obtained have been compared with those obtained by other well known classification techniques, included a standard LVQ. The data set at hand has been divided in a training and a test set. The former set was used to train both our system and the other classifiers taken into account for the comparison. As regards our system, once a set of prototypes has been obtained, the classification of an unknown patterns of the test set is performed by assigning to it the label of the nearest prototype in the feature space. The experiments performed by using the data taken into account have shown very interesting results and have confirmed the effectiveness of the proposed approach.

The remainder of the paper is organized as follows: in section 2 a formalization of data classification is described; section 3 illustrates the standard LVQ algorithm, while in section 4 the proposed approach is detailed. In section 5 the experimental results are presented, while section 6 is devoted to the conclusions.

\section{Data Classification}

In the data classification context a set of objects to be analyzed is called data set, and each object is called pattern and represented by $\mathbf{X}=\left(x_{1}, \ldots, x_{\ell}\right)$ with $\mathbf{X} \in \mathbf{S}$, where $\mathbf{S}$ is the universe of all possible elements characterized by $\ell$ features and $x_{i}$ denotes the $i$-th feature of the pattern. A data set with cardinality $N_{D}$ is denoted by $\mathcal{D}=\left\{\mathbf{X}_{1}, \ldots, \mathbf{X}_{N_{D}}\right\}$ with $\mathcal{D} \subseteq \mathbf{S}$. The set $\mathcal{D}$ is said labeled if it exists a set of integers:

$$
\Lambda=\left\{\lambda_{1}, \ldots, \lambda_{N_{D}}\right\}: \lambda_{i} \in[1, c]
$$

The $i$-th element $\lambda_{i}$ of $\Lambda$ is said the label of the $i$-th pattern $\mathbf{X}_{i}$ of $\mathcal{D}$. We will say that the patterns of $\mathcal{D}$ can be grouped into $c$ different classes. Moreover, given the pattern $\mathbf{X}_{i}$ and the label $\lambda_{i}=j$, we will say that $\mathbf{X}_{i}$ belongs to the $j$-th class.

Given a data set $\mathcal{D}=\left\{\mathbf{X}_{1}, \ldots, \mathbf{X}_{N_{D}}\right\}$ containing $c$ classes, a classifier $\Gamma$ is defined as a function

$$
\Gamma: \mathcal{D} \longrightarrow[0, c]
$$

In other words, a classifier assigns a label $\gamma_{i} \in[0, c]$ to each input pattern $\mathbf{X}_{i}$. If $\gamma_{i}=0$, the corresponding pattern $\mathbf{X}_{i}$ is said rejected. This fact means that the classifier is unable to trace the pattern back to any class.

The pattern $\mathbf{X}_{i}$ is recognized by $\Gamma$ if and only if:

$$
\gamma_{i}=\lambda_{i}
$$

otherwise the pattern is said misclassified. If $N_{\text {corr }}$ is the number of patterns of $\mathcal{D}$ recognized by $\Gamma$ the ratio $N_{\text {corr }} / N_{D}$ is defined as the recognition rate of the classifier $\Gamma$ obtained on the data set $\mathcal{D}$. 


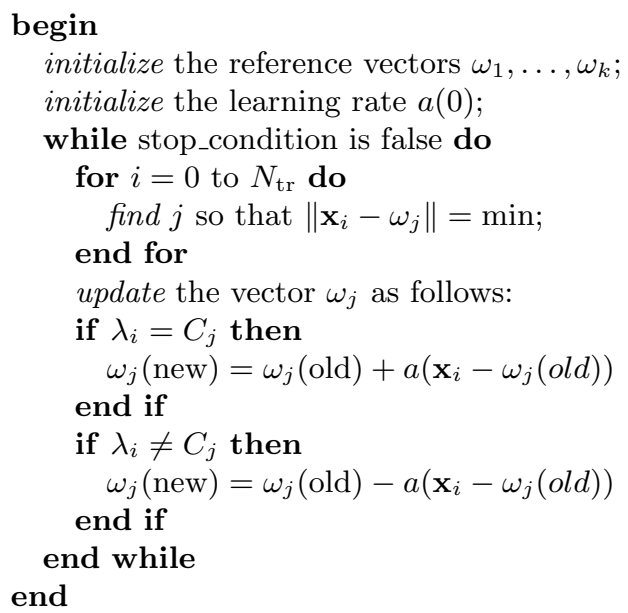

Fig. 1. The algorithm used in order to determine the position of the reference vectors in the feature space. $N_{\mathrm{tr}}$ is the number of patterns in the training set, while $\lambda_{i}$ and $C_{j}$ respectively represent the labels of the pattern $\mathbf{x}_{i}$ and of the "winner" vector $\omega_{j}$. This algorithm is often referred in the literature to as "the winner take all" (WTA). Note that usually the stop_condition specifies a certain number of iterations (epochs in the LVQ jargon)

\section{Learning Vector Quantization}

The Learning Vector Quantization (LVQ) methodology was introduced by Kohonen [12] and in the last years has widely been used to perform classification tasks. LVQ classifiers have been applied in a variety of practical problems, such as medical image analysis, classification of satellite spectral data, fault detection in technical processes, and language recognition [13]. The LVQ approach offers a method to form a quantized approximation of an input data set $\mathcal{D} \subset R^{p}$ using a finite number $k$ of reference vectors $\omega_{i} \in R, i=1, \ldots, k$. After that an LVQ has been trained, the feature space in which the data are represented, is partitioned with a Voronoi tessellation. This tessellation partitions the feature space into a certain number of disjoint regions, each identified by a different reference vector. These regions are bordered by hyperplanes defined as the loci of points that are equidistant from the two nearest reference vectors. Hence, a reference vector represents the prototype of the points inside the corresponding regions.

Given an incoming pattern $\mathbf{x}$ to be recognized and a set of reference vectors $\left\{\omega_{1}, \ldots, \omega_{k}\right\}$, the classification is performed by computing the Euclidean distance between $\mathbf{x}$ and each of the reference vector $\omega_{i}$. The pattern is then assigned to the closest reference vector and is recognized as belonging to the same class of the reference vector to which it has been assigned. In the LVQ method the 


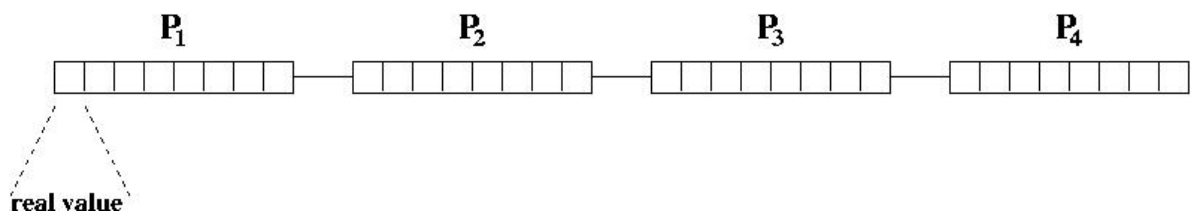

Fig. 2. An example of individual containing 4 prototypes. In this example, the prototypes are vectors in a 8-dimensional space.

reference vectors are a priori labeled and algorithmically determined by using a training set of labeled patterns (see figure 1). Note that, usually, the number of reference vectors for each of the classes have to be provided by the user.

\section{Prototype Generation}

As mentioned above, the prototypes to be used for classification are represented by points in the feature space. The method proposed in this paper for finding a good set of prototypes is based on a particular class of genetic algorithms [2], namely the Breeder Genetic Algorithms (BGA) [14], in which the individuals are encoded as real valued vectors. In our case, an individual consists of a variable length list of feature vectors, each one representing a prototype (see Figure 2).

The system, accordingly to the EA paradigm, starts by generating a population of $\mathcal{P}$ individuals. The number of prototypes (in the following referred as length) of these initial individuals is randomly assigned in the range $\left[N_{\min }, N_{\max }\right]$. Each prototype is initialized by randomly choosing a pattern in the training set. Afterwards, the fitness of the individuals generated is evaluated. A new population is generated in two ways: on one side, according to an elitist strategy, the best $\mathcal{E}$ individuals are selected and just copied. On the other side, $(\mathcal{P}-\mathcal{E}) / 2$ couples of individuals are selected by using a selection mechanism. The crossover operator is then applied to each of the selected couples, according to a chosen probability factor $p_{c}$. The mutation operator is then applied to the individuals according to a probability factor $p_{m}$. Finally the obtained individuals are added to the new population. The process just described is repeated for $N_{g}$ generations.

The fitness function, the selection mechanism and the operators employed are described in the following.

\subsection{Fitness Function}

Each individual is evaluated using a training set $\mathcal{D}_{t r}$ containing $N_{\text {tr }}$ patterns. This evaluation implies the following steps:

1. Every pattern in the training set is assigned to the nearest prototype (i.e. reference vector) in the individual to be evaluated. Euclidean distance is used in the feature space. After this step, $n_{i}\left(n_{i} \geq 0\right)$ patterns will have 


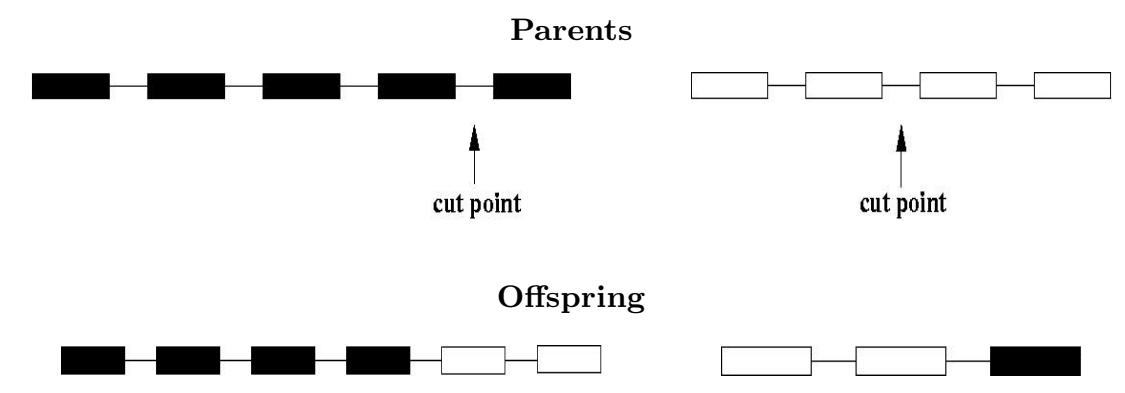

Fig. 3. An example of application of the crossover operator.

been assigned to the $i$-th prototype. The prototypes for which $n_{i}>0$ will be referred in the following as valid. The prototypes for which $n_{i}=0$ will be ignored in the following steps.

2. Each valid prototype of an individual is labeled with the label most widely represented in the corresponding cluster.

3. The recognition rate obtained on $\mathcal{D}_{t r}$ is computed and assigned as fitness value to the individual.

In order to favor the individuals able to obtain good performances with a lesser number of prototypes, the fitness of each individual is increased by $0.1 / N_{p}$, where $N_{p}$ is the number of prototypes in an individual. Moreover, the individuals having a number of prototypes out of the interval $\left[N_{\min }, N_{\max }\right]$ are killed, i.e. marked in such a way that they are not chosen by the selection mechanism.

\subsection{Selection Mechanism}

The tournament method has been chosen as selection mechanism. In the tournament selection, a number $\mathcal{T}$ of individuals is chosen randomly from the population and the best individual from this group is selected as parent. This process is repeated for as many individuals have to be chosen. Such a mechanism ensures to control the loss of diversity and the selection intensity [15].

\subsection{Genetic Operators}

In the approach presented here two genetic operators have been devised: crossover and mutation. The crossover operator belongs to the wider class of recombination operators: it accepts in input two individuals and it yields as output two new individuals. This operator acts at list level and gives our system the important feature of automatically discovering the number of prototypes actually 


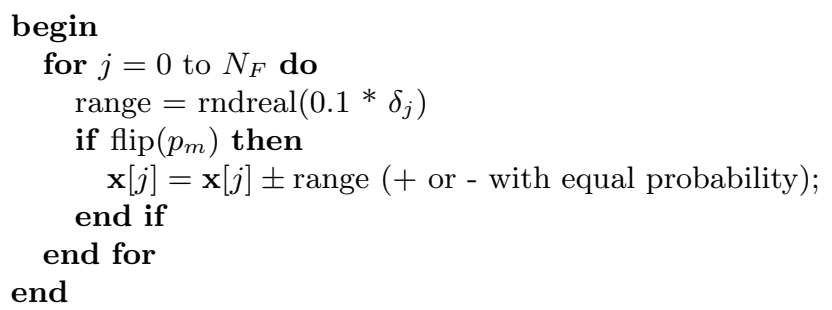

Fig. 4. The mutation operator applied to each of the prototypes, i.e. reference vectors, in an individual. $N_{F}$ is the number of features of the patterns in the data analyzed. $\delta_{j}$ is the range of the $j$-th feature computed on the training set, while $p_{m}$ represents the probability of mutation of each single feature value in the prototype.

needed to represent the classes defined in the problem at hand. The mutation operator, instead, manipulates a single individual. Its effect is that of "moving" the prototypes (i.e. vectors) of the input individual in the feature space. These operators are detailed in the following.

Crossover. The crossover operator is applied to two individuals $I_{1}$ and $I_{2}$ and yields two new individuals by swapping parts of the lists of the initial individuals, without breaking any single prototype. Assuming that the length of $I_{1}$ and $I_{2}$ are respectively $l_{1}$ and $l_{2}$, the crossover is applied in the following way: the first individual is split in two parts by randomly choosing an integer $t_{1}$ in the interval $\left[1, l_{1}\right]$. The obtained lists of vectors $I_{1}^{\prime}$ and $I_{1}^{\prime \prime}$ will have length $t_{1}$ and $l_{1}-t_{1}$ respectively. Analogously, by randomly choosing an integer $t_{2}$ in the interval $\left[1, l_{2}\right]$, two lists of prototypes $I_{2}^{\prime}$ and $I_{2}^{\prime \prime}$, respectively of length $t_{2}$ and $l_{2}-t_{2}$, are obtained from $I_{2}$. At this stage, in order to obtain a new individual, the lists $I_{1}^{\prime}$ and $I_{2}^{\prime \prime}$ are merged. This operation yields a new individual of length $t_{1}+l_{2}-t_{2}$. The same operation is applied to the remaining lists $I_{2}^{\prime}$ and $I_{1}^{\prime \prime}$ and a new individual of length $t_{2}+l_{1}-t_{1}$ is obtained. The number of the swapped prototypes depends on the integers $t_{1}$ and $t_{2}$. An example of application of this operator is given in figure 3. As mentioned above the implemented crossover operator allows one to obtain offspring individuals whose length may be quite different from that of the parents. As a consequence, during the evolution process, individuals made of a variable number of prototypes can be evolved.

Mutation. Given an individual $I$, the mutation operator is independently applied to each prototype of $I$. In figure 4 the algorithm used to modify each of the prototypes in an individual is shown. 


\begin{tabular}{lcc} 
Parameter & symbol & value \\
\hline Population size & $\mathcal{P}$ & 300 \\
Tournament size & $\mathcal{T}$ & 6 \\
elithism size & $\mathcal{E}$ & 5 \\
Crossover probability & $p_{c}$ & 0.4 \\
Mutation probability & $p_{m}$ & 0.05 \\
Number of Generations & $N_{g}$ & 500
\end{tabular}

Table 1. Values of the basic evolutionary parameters used in the experiments.

\section{$5 \quad$ Experimental Results}

In order to ascertain its effectiveness, the proposed approach has been tested on data extracted from two landsat 6 band multispectral images. The first one ${ }^{4}$ is 2030x1167 pixels large and has been taken in order to distinguish between forestnon forest areas, while the second one ${ }^{5}$ is a $1000 \times 1000$ pixels large, related to the land cover mapping for desertification studies. To this images a segmentation method has been applied in order to obtain regions formed by the same type of pixel [16]. For each of the region provided by the segmentation, a set of features have been extracted, related to its geometrical characteristics and to its spectral data. For each region the extracted features have been used to build up a data record.

From the first image a data set made up of 2500 items, i.e. regions, have been derived; each item may belong to one of two classes, forest or non-forest. From the second image 7600 items have been extracted and the items may belong to 7 classes, representing various land cover types: various vegetation or water. Although the segmentation process used extracts more than 10 features for each of the regions identified, for both the images analyzed only six features have been considered.

Preliminary trials have been performed to set the basic evolutionary parameters reported in Table 1. This set of parameters has been used for all the experiments reported in the following. Since our approach is stochastic, as well as all the EC-based algorithms, 20 runs have been performed for each data set taken into account. The reported results are those obtained using the individual having the highest fitness among those obtained during the 20 performed runs.

The results obtained by our method on the data described above have been compared with those obtained by other three classification algorithms: nearestneighbor $(\mathrm{NN}), k-\mathrm{NN}$ and a standard LVQ. These algorithms are detailed in the following:

\footnotetext{
${ }^{4}$ The image has been provided by courtesy from JRC.

5 This image has been provided by courtesy from ACS spa as part of the Desert Watch project.
} 


\begin{tabular}{|c|c|c|c|c|}
\hline & NN & 9-NN & LVQ & EC-LVQ \\
\hline Mean & 72.69 & 83.05 & 72.05 & 82.50 \\
\hline Std & 4.34 & 0.25 & 3.36 & 1.7 \\
\hline N $_{\mathbf{P}}$ & 2250 & 2250 & 80 & $10.6(1.7)$ \\
\hline
\end{tabular}

Table 2. Means and standard deviations of recognition rate on the test set for the forest cover dataset.

\begin{tabular}{|c|c|c|c|c|}
\hline & NN & 6-NN & LVQ (700) & EC-LVQ \\
\hline Best & 69.2 & 74.08 & 76.47 & 75.6 \\
\hline $\mathbf{N}_{\mathbf{P}}$ & 3800 & 3800 & 700 & 136 \\
\hline
\end{tabular}

Table 3. The recognition rates on the test set obtained for the land cover dataset.

LVQ. The LVQ used for the comparison of our results is an improved version of that described in section 3, it is called Frequency Sensitive Competitive Learning (FSCL) [17] and is often used to compare the performances of other algorithms.

Nearest Neighbor. Let $\mathcal{D}_{\text {tr }}$ be a training set of $N_{\text {tr }}$ labeled patterns represented by feature vectors. A nearest neighbor (NN) classifier recognizes an unknown pattern $\mathbf{x}$ by computing the Euclidean distance between $\mathbf{x}$ and each of the patterns in $\mathcal{D}_{\mathrm{tr}}$. Then $\mathbf{x}$ is recognized as belonging to the same class of the nearest pattern in $\mathcal{D}_{\mathrm{tr}}$. It has been shown that a NN classifier does not guarantee the minimum possible error, i.e. the Bayes rate [1].

$\boldsymbol{k}$-Nearest Neighbor. A $k$-Nearest Neighbor $(k$-NN) classifier is an extension of the NN classifier described above. In fact, given a training set $\mathcal{D}_{\mathrm{tr}}$, a pattern $\mathbf{x}$ to be recognized is attributed to the most widely represented class among the $k$ nearest patterns of $\mathcal{D}_{\mathrm{tr}}$. In the experiments reported in the following the number of neighbors has been varied between 1 and 15 .

\subsection{Comparison Findings}

In Table 2 the results obtained on the forest cover data set are shown. In all the runs performed on this data set the minimum length $N_{\min }$ allowed for an individual has been set to 2 , while the maximum one has been set to 20 . In order to avoid any bias in the comparison, due to the low number of patterns in the data set, the 10 fold cross validation procedure has been adopted. In this procedure, the performances of a classifier on a data set $\mathcal{D}$ are evaluated by randomly dividing $\mathcal{D}$ into 10 disjoint sets of equal size $N / 10$, where $N$ is the total number of patterns in $\mathcal{D}$. Then the classifier is trained 10 times, each time with a different set held out as a test set. In order to evaluate the performance 


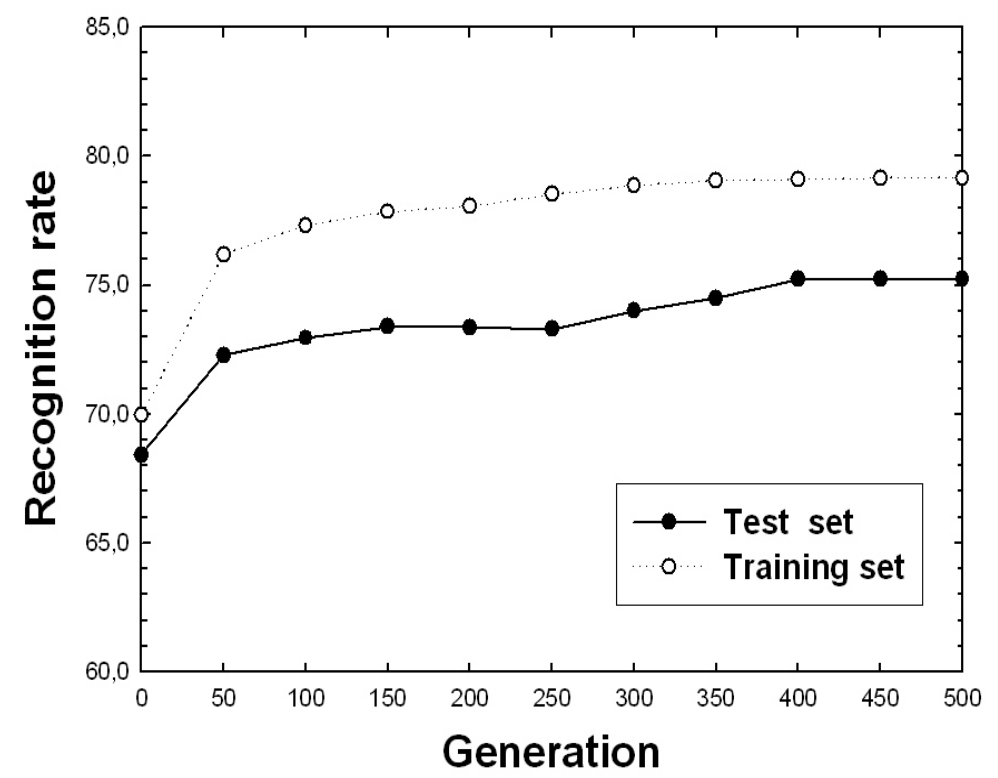

Fig. 5. Recognition rate on training and test sets for the land cover data set during the best run.

of the classifier on unknown data, the performance is computed as the mean of the results obtained on the ten different test sets [1] As a consequence of the choice of this procedure, 200 runs have been performed.

In Table 2, the mean and the standard deviation obtained on the 10 test sets are reported together with the number of prototypes employed are reported. As regards the $\mathrm{NN}$ and the $k-\mathrm{NN}$ classifiers, the number of prototypes equals the number of patterns in the training set, while for the LVQ this number has been set to 80. For our EC-based LVQ method, this number is not provided by the user, but it has been automatically found by the implemented system. Particularly, the average number of prototypes, found for the ten considered test set, equals to 10.6 , while the standard deviation is 1.7 . Thus the automatism devised allow the system to strongly reduce the number of prototypes needed to perform the classification task demanded. The outcome of this reduction is a strong enhancement of the classifier efficiency. As regards the recognition rate obtained on test set by the different algorithms, the performance of our system is significantly better than that of the NN and LVQ classifiers, although that of the $k$-NN (obtained setting $k$ equal to 9) are slightly better than that of our system. However, in our opinion, the huge difference in the number of necessary prototypes used compensates this little difference in the recognition rate.

In Table 3 the results obtained on the land cover data set are shown. In this case the original data set has been randomly split in two sets, respectively as 
training set and test set. For this data set the total number of executed runs has been 20. In Table 3 the best results obtained and the number of prototypes employed are reported. Our results are significantly better than those obtained form the NN classifier and slightly better than that obtained from the $k-\mathrm{NN}$ (in this case $k$ has been set equal to 6). Only the LVQ classifier has obtained a performance slightly better than ours, but such performance has been obtained with a total number of 700 prototypes, while our performance has been obtained using only 136 prototypes. Also in this case, the difference in the number of prototypes compensates the little difference in the performance achieved.

In a learning process, in most cases, when the maximum performance is achieved on training set, the generalization power, i.e. the ability of obtaining similar performance on unknown data (the test set), may significantly decrease. In order to investigate such aspect for our system, the recognition rates on training and test set have been taken into account for the different considered data sets. In Figure 5 such recognition rates, evaluated every 50 generations, in the best run for the desert data set, are displayed. It can be observed from the figure that, in the experiments carried out, the recognition rate increases with the number of generations both for the training set and for the test set. The best recognition rates occur in both cases nearby generation 400. Moreover, the fact that the difference between the two recognition rates does not tend to increase when that on the training set reaches its maximum, demonstrates the good generalization power of our system.

\section{Conclusions and Feature Work}

A new method that uses the evolutionary computation paradigm has been devised for generating prototypes for a LVQ-based classifier. The patterns belonging to the different classes, represented as vectors in a feature space, are represented by prototypes obtained by evolving an initial population of randomly selected feature vectors. The devised approach does not require any a priori knowledge about the actual number of prototypes needed to represent the classes defined in the problem at hand. The method has been tested on satellite images and the results have been compared with those obtained by other classification methods. The obtained results and the comparisons performed have confirmed the effectiveness of the approach and outlined the good generalization power of the proposed method.

The results could be easily improved by applying the mutation operator in a way that takes into account the performances obtained by the single prototypes. In practice, the probability of application of the mutation operator to a single prototype should be computed as a function of its performance. Specifically, the lower is the recognition rate obtained by the prototype, the lower should be the probability of applying of the mutation to it. In this way, the research of prototypes becomes more effective, since the probability of modifying "good" prototypes is much lower than that of modifying "bad" prototypes, i.e. those performing worse in recognizing patterns belonging to the same class. 


\section{Acknowledgments}

The authors gratefully acknowledge the support of the Joint Research Centre (JRC) that supplied the forest cover data and the ACS spa which supplied the land mapping data as part of the Desert Watch project.

\section{References}

1. Duda, R.O., Hart, P.E., Stork, D.G.: Pattern Classification. John Wiley \& sons, Inc. (2001)

2. Goldberg, D.E.: Genetic Algorithms in Search Optimization and Machine Learning. Addison-Wesley (1989)

3. Lanzi, P.L., Stolzmann, W., Wilson, S.W., eds.: Learning Classifier Systems: From Foundations to Applications. Volume 1813 of Lecture Notes on Artificial Intelligence. Springer-Verlag, Berlin, Germany (2000)

4. Giordana, A., Neri, F.: Search-intensive concept induction. Evolutionary Computation 3 (1995) 375-416

5. Greene, D.P., Smith, S.F.: Competition-based induction of decision models from examples. Machine Learning (1993) 229-257

6. Janikow, C.Z.: A knowledge-intensive genetic algorithm for supervised learning. Machine Learning (1993) 189-228

7. De Jong, K.A., Spears, W.M., Gordon, D.F.C., Janikow, Z.: Using genetic algorithms for concept learning. Machine Learning (1993) 161-188

8. Agnelli, D., Bollini, A., Lombardi, L.: Image classification: an evolutionary approach. Pattern Recognition Letters 23 (2002) 303-309

9. Rauss, P.J., Daida, J.M., Chaudhary, S.A.: Classification of spectral image using genetic programming. In: Genetic and Evolutionary Computation Conference (GECCO). (2000) 726-733

10. Kishore, J.K., Patnaik, L.M., Mani, V., Agrawal, V.K.: Application of genetic programming for multicategory pattern classification. IEEE Transactions on Evolutionary Computation 4 (2000) 242-258

11. Mendes, R., Voznika, F., Freitas, A., Nievola, J.: Discovering fuzzy classification rules with genetic programming and co-evolution. In: Principles of Data Mining and Knowledge Discovery (Proc. 5th European Conference PKDD 2001) - Lecture Notes in Artificial Intelligence. 2168, Berlin, Springer-Verlag (2001) 314-325

12. Kohonen, T.: Self-Organizing Maps. 3-rd edn. Springer-Verlag New York, Inc., Secaucus, NJ, USA (2001)

13. Karayiannis, N.B.: Learning vector quantization: A review. International Journal of Smart Engineering System Design 1 (1997) 33-58

14. Muhlenbein, H., Schlierkamp-Voosen, D.: The science of breeding and its application to the breeder genetic algorithm (bga). Evolutionary Computation 1 (1993) 335-360

15. Blickle, T., Thiele, L.: A comparison of selection schemes used in genetic algorithms. Technical Report 11, Swiss Federal Institute of Technology (ETH), Gloriastrasse 35, 8092 Zurich, Switzerland (1995)

16. D'Elia, C., Poggi, G., Scarpa, G.: A tree-structured random markov field model for bayesian image segmentation. IEEE Transactions on Image Processing 12 (2003) 1259-1273

17. Ahalt, S., Krishnamurthy, A., Chen, P., Melton, D.: Competitive learning algorithms for vector quantizationn. Neural Networks 3 (1990) 277-290 